\title{
Exploring the Environment of the most powerful Explosions
}

\author{
Antonino Cucchiara ${ }^{1}$, Mark Rafelski ${ }^{1}$, Michele Fumagalli ${ }^{2,3}$, \\ Daniel Kocevski ${ }^{1}$, Jason X. Prochaska ${ }^{4}$, Ryan J. Cooke ${ }^{4}$ \\ and G. D. Becker ${ }^{5}$ \\ ${ }^{1}$ NASA Postdoctoral Program Fellow, Goddard Space Flight Center, \\ Greenbelt, MD 20771, USA, \\ email: antonino.cucchiara@nasa.gov \\ ${ }^{2}$ Institute for Computational Cosmology, Department of Physics, Durham University, \\ South Road, Durham, DH1 3LE, UK \\ ${ }^{3}$ Carnegie Observatories, 813 Santa Barbara Street, Pasadena, CA 91101, USA \\ ${ }^{4}$ Department of Astronomy and Astrophysics, UCO/Lick Observatory, University of \\ California, 1156 High Street, Santa Cruz, CA 95064, USA \\ ${ }^{5}$ Kavli Institute for Cosmology and Institute of Astronomy, University of Cambridge, \\ Madingley Road, Cambridge, CB3 0HA, UK
}

\begin{abstract}
More than 60 GRBs at $z \gtrsim 1.5$ reside in the vicinity of dense, cold gas as probed by the measured neutral hydrogen via afterglow absorption spectroscopy. We present the largest sample of GRB-DLAs to date in comparison with a sample of DLAs along quasars: the metallicity of the GRB hosts represents a unique tool to understand if this particular subset of galaxies can be the key ingredient for GRB formation (and massive stars) at any redshift as well as the overall cosmic star-formation rate. We show that GRB-DLAs live in a metal enriched environment, especially at $z \gtrsim 4$, likely the result of recent intense star formation and/or SNe episodes. We also derive that our metallicity measurements are broadly consistent with a mild metallicity bias for the GRB formation.
\end{abstract}

Keywords. gamma rays: bursts, galaxies: ISM, ISM: abundances

\section{Introduction}

The increased number of Gamma-ray Bursts (GRB) with redshift discovered during the last 10 years, thanks to the Swift satellite, has given us a new view of the highredshift Universe exploration. We have now 130 GRBs with afterglow spectra and a similar number of host galaxies spectra in the redshift range $z=0-8$. These studies, in combination of host galaxy surveys (Perley 2013, Hjorth 2012, Krühler 2015) performed from ground and space based facilities, have confirmed that GRBs are likely linked with the cosmic star-formation, they provide location for the small, blue and star-forming galaxies that recognized the Universe during reionization, and they can serve as tracers of metal production throughout cosmic time.

\section{GRBs and Interstellar medium}

Absorption spectroscopy of GRB afterglows have been used, similarly to quasars to study the properties of material along the line of sight ("intervening systems"), in particular the ones that show strong Lyman-alpha absorption features (at $1216 \AA$ rest-frame). This absorption line, differently than in QSO spectra, arise at the same redshift than the 


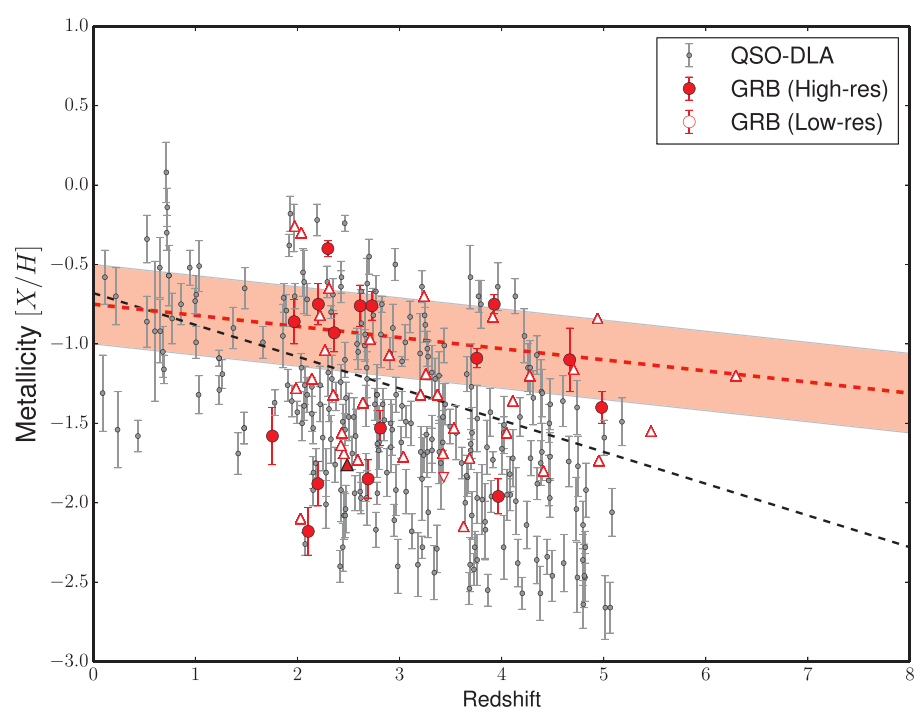

Figure 1. Path of pre-solar grains from their stellar sources to the laboratory.

GRBs, providing direct evidence of the presence of a so-called Damped Lyman-alpha system (DLA) in the GRB host. DLAs are important because they harbor the cold matter (hydrogen and low-ionized metals) that fuel star-formation. A key advantage of GRBs is the their simple intrinsic afterglow spectrum (a single power-law compared to the more complex QSO ones) and their brightness, which makes them detectable up to the highest redshift.

Several types of absorption features have been identified, from low-ionized material (like CII,SiII) as well as high-ionized transitions (CIV,SiIV) and fine-structure transitions (FeII*,NiII*) which trace clouds of material very close to the GRB source of radiation.

We collected 56 GRB-DLA spectra and measured absorption line metallicity consistently using sulphur low-ionized lines which are proved to be less sensitive to metallicity Cucchiara 2015. We compared then our findings with the largest sample available of QSO-DLAs (DLAs found along the line of sight of quasars. Our main result can be seen in Figure 1 and it is twofold: 1 ) in the redshift range $z=1.5-3$ GRBs- and QSO-DLAs share the same parameter space in metallicity supporting the idea that the galaxies that host DLAs are similar and that the line of sight intersects randomly metal rich as well as metal poor clouds of gas; 2 ) at $z \gtrsim 3$, instead GRBs seem to probe my higher metal content than QSO-DLAs, which instead probe more metal poor regions of galaxies. This may indicate that GRB-DLAs and GRB progenitor itself may live in regions of their hosts where metal have been produced already (by AGB stars or supernovae).

Clearly more data are needed, in particular at the highest redshifts, but this work prove once more how only GRBs can provide insight of the in-situ star formation at such early time.

\section{References}

Perley, D. A. et al. 2013 ApJ, 778, 128

Hjorth, J. et al. 2012 ApJ, 756, 187

Krühler, T. et al. 2015 A\&A, 581, 125

Cucchiara, A. et al. 2015 ApJ, 804, 51 